\title{
Light-Based Devices for the Treatment of Facial Erythema and Telangiectasia
}

\author{
Vani Yepuri · Anant D. Patil · Klaus Fritz • Carmen Salavastru • \\ George Kroumpouzos • Steven Paul Nisticò - Domenico Piccolo • \\ Ahmed Sadek · Ashraf Badawi - Martin Kassir • Michael H. Gold • \\ Stephan Große-Büning · Stephan Grabbe · Mohamad Goldust
}

Received: June 21, 2021 / Published online: September 25, 2021

(C) The Author(s) 2021

\section{ABSTRACT}

Facial erythema is one of the most common outpatient complaints in dermatology. There are various causes of facial erythema and several devices are available for its treatment. Pulsed dye laser (PDL) and intense pulsed light (IPL) are the two common light devices used for these conditions. In this review, we evaluated the literature to assess efficacy of IPL versus PDL in

\section{Yepuri}

Venkat Charmalaya, Centre for Advanced Dermatology and Post Graduate Training, Bangalore, Karnataka, India

\section{A. D. Patil}

Department of Pharmacology, Dr. DY Patil Medical College, Navi Mumbai, India

\section{K. Fritz}

Dermatology and Laser Center, Reduitstr. 13, 76829 Landau, Germany

K. Fritz · C. Salavastru

"Carol Davila" University of Medicine and Pharmacy, 37 Dionisie Lupu Str, 020021 Bucharest, Romania

C. Salavastru

"Colentina" Clinical Hospital, Pediatric Dermatology Discipline, Dermato-Oncology Research Facility, 1921 Stefan cel Mare Str, Bucharest, Romania

G. Kroumpouzos

Department of Dermatology, Alpert Medical School of Brown University, Providence, Rhode, Island facial erythema and telangiectasia. We searched published articles including clinical trials or reviews articles, case series, and case reports. Electronic databases (MEDLINE and PubMed) were searched to retrieve the articles. Reference lists of selected articles were also considered for the review. Articles published in English language until June 2021 were considered for this review.

\section{G. Kroumpouzos}

Department of Dermatology, Medical School of Jundiaí, São Paulo, Brazil

G. Kroumpouzos

GK Dermatology, PC, South Weymouth, Massachusetts, USA

\section{S. P. Nisticò}

Dermatology Unit, Department of Health Sciences, Magna Graecia University of Catanzaro, 88100 Catanzaro, Italy

D. Piccolo

Skin Center-Dermo Aesthetic Laser Centers, Avezzano, Italy

\section{A. Sadek}

Cairo Hospital for Dermatology and Venereology (Al-Haud Al-Marsoud) Manager, Cairo, Egypt

\section{A. Badawi}

Medical Laser Applications, National Institute of Laser Enhanced Sciences, Cairo University, Giza, Egypt 
Keywords: Facial erythema; Efficacy; Purpura; Teleangiectasia

\section{Key Summary Points}

Facial erythema and telangiectasia, in general, respond poorly to treatment

Both intense pulsed light and pulsed dye laser are effective in treating erythema

Intense pulsed light offers some advantages such as larger spot size, fewer adverse events, and longer treatment maintenance time

More studies are needed to assess the effectiveness of current treatment options in facial erythema and telangiectasia

\section{INTRODUCTION}

Facial erythema is a common condition with which patients of Fitzpatrick's skin type I and II visit dermatologists. Many times, facial erythema is physiological and transient in nature, appearing after sudden emotions, exercise, or

\author{
A. Badawi \\ Dermatology and Allergology Department, Faculty \\ of Medicine, Szeged University, Szeged, Hungary \\ A. Badawi \\ Oakville, Canada \\ M. Kassir \\ Worldwide Laser Institute, Dallas, USA \\ M. H. Gold \\ Gold Skin Care Center, Tennessee Clinical Research \\ Center, Nashville, TN, USA \\ S. Große-Büning · M. Goldust ( $\square)$ \\ Department of Dermatology, University Medical \\ Center Mainz, Mainz, Germany \\ e-mail: mgoldust@uni-mainz.de \\ S. Grabbe \\ Department of Dermatology, University Medical \\ Center of the Johannes Gutenberg University, \\ Mainz, Germany
}

exposure to heat, whereas various diseases may result in longer-lasting erythema [1-3]. Rosacea is a common and chronic inflammatory disorder associated with transient central facial erythema that sometimes becomes persistent because of recurrent episodes [4]. Facial erythema may be a symptom of other skin diseases like lupus erythematodes, dermatomyositis, drug eruptions, or systemic bacterial infection.

Proper evaluation of facial erythema to establish cause and diagnosis will help in assessing the type of treatment required to reduce the redness. Use of energy and laser/ light-based therapies has significantly increased in dermatology practice over the years. These include pulsed dye laser (PDL), intense pulse light (IPL), KTP (532 nm), yellow laser $(577 \mathrm{~nm})$, and sometimes $1064 \mathrm{~nm}$ long pulsed with small spots. The objective of this review was to evaluate role of light-based devices in the treatment of facial erythema and telangiectasia.

\section{METHODS}

A comprehensive literature search was performed to retrieve and select articles related to IPL and PDL in facial erythema and telangiectasia published in MEDLINE/PubMed and Google Scholar. Relevant references from the selected articles were also referred for review. Articles published in English until April 2021 were considered for this review. All types of clinical studies (clinical trials, observational studies, retrospective studies), case series, and case reports were selected. Animal studies were also considered for the review. Duplicate studies retrieved from two databases were removed.

This article is based on previously conducted studies and does not contain any new studies with human participants or animals performed by any of the authors.

\section{RESULTS AND DISCUSSION}

\section{Role of Laser in Facial Erythema}

Vascular lasers are often used by cosmetic dermatologists for reducing facial redness. 
Introduction of laser devices dates back to the 1960 s, but it was only in 1980s that lasers with specific targets were innovated, one among them being vascular lasers $[5,6]$. The lasers used to treat vascular lesions emit a wavelength which targets specifically the oxyhemoglobin. Oxyhemoglobin absorbs the laser and then it is converted to heat, which further causes heat damage to the Lamina intima of the vessel and consequently vessel wall coagulation and vessel obliteration [7]. These vascular lasers cause heating within dermal blood vessels, resulting in reduction of the diameter of blood vessels [8]. Three aspects which play an important role in providing effective results with lasers include wavelength, pulse duration, and fluence [9]. Multiple laser systems with different wavelengths are shown to be effective in the treatment of vascular lesions.

\section{PDL}

This highly effective treatment for vascular lesions works on the principle of selective photothermolysis and is used in wide range of conditions including port-wine stains, facial telangiectasia, hemangiomas, redness of scars, pyogenic granulomas, Kaposi's sarcoma, and poikiloderma of Civatte [10-13].

In some studies combination of oxymetazoline and PDL has been evaluated. In an animal study, Kelly and colleagues compared the effects of oxymetazoline with PDL combination and found it to be more effective than saline plus PDL [14]. In a retrospective study, Suggs et al. reported effective reduction of erythema and telangiectasias after combination treatment with PDL plus oxymetazoline $1.0 \%$ cream [15].

Clark and colleagues assessed 12 patients and demonstrated that PDL is a safe and effective treatment for the erythematotelangiectatic component of rosacea. However, there may be difficulty in clearing more intense erythema resulting in discomfort or itching and conspicuous teleangiectatic vessels on the nose [16]. In a study involving ten patients with idiopathic flushing, 585-nm PDL was a safe and efficacious treatment with significant improvement in the erythema by more than $62 \%$ [17]. In a double- blinded, split-face randomized controlled trial diffuse facial erythema was treated with nonpurpuragenic 595-nm PDL or microsecond 1064-nm Nd:YAG laser. Both lasers were found to be efficacious, though PDL may be more effective and Nd:YAG was less painful [18]. In facial erythema due to acne, nonablative, $1550-\mathrm{nm}$ fractional laser and 595-nm PDL treatments were effective and well tolerated. More patients reported good or excellent results on the fractional laser-treated sites than PDL treatment (91.7\% vs 75\%) [19]. In another splitface comparative study $(n=15), 595-n m$ PDL and a pulsed 532-nm KTP laser were effective in the treatment of facial telangiectasia and redness with the latter being more effective and associated with higher rates of side effects [20].

In a prospective study among patients with facial telangiectasia, flashlamp PDL-treated areas had excellent results compared with argon tunable dye laser with robotized handpiece $(\mathrm{ATDL} / \mathrm{H})$ [21]. Ultra-long pulse width pulsed dye laser and elliptical spot can be effective in patients with telangiectasia resistant to KTP laser and PDL [22]. Spider telangiectasia treated by the flashlamp-pumped PDL has been reported to have excellent results in most patients after a single treatment without any permanent sequelae [23].

A small study also showed effectiveness of PDL for skin lesions due to hereditary hemorrhagic telangiectasia. Excellent clearance was seen after a mean of 2.6 treatments [24]. In another small study, an average of about three courses of treatment with Chromos PDL $(585 \mathrm{~nm})$ was effective in the treatment of hemorrhagic telangiectasias in the anterior nasal cavity [25].

\section{Adverse Events with PDL and Their Prevention or Treatment}

Flashlamp PDL is better in terms of efficacy than ATDL. However, as a result of purpura and postinflammatory hyperpigmentation, patients may not prefer flashlamp PDL [26]. Multi-pass treatment with extended pulse width $(40 \mathrm{~ms})$ may be effective without producing purpura. Increasing the threshold for purpura can allow 
use of higher fluences [27]. Impressive results in facial telangiectasia can be seen after a single purpura-free treatment with the variable-pulse PDL, but improvement may be greater after induction of purpura in thicker telangiectasia. Such treatment is also useful in some telangiectasia without resultant bruising [28]. Cold air cooling can be useful to reduce the incidence of side effects and improve patient satisfaction in facial telangiectasia treatment. Cooling based on patients' tolerance and increasing energy density may improve the results [29]. Other adverse effects of laser therapy include transient hyperpigmentation, vesicle formation, and scarring. It is important to avoid damage to the surrounding tissue while using laser therapy.

\section{Long-Pulsed Nd:YAG}

Long-pulsed Nd:YAG and PDL are both effective in rosacea-associated nasal telangiectasia [30] but should be used carefully to avoid side effects. Nd:YAG penetrates deeper than PDL with a higher potential for destroying tissue due to a higher amount of energy and heat. Longpulsed Nd:YAG may be used with low fluence and small spot sizes for superficial penetration. Always make sure that proper cooling is available before, during, and after the procedure.

\section{IPL}

The first IPL system was introduced in the mid1990s [6]. This high-powered broadband light source has various applications in dermatology practice. It has been commonly used in treatment of facial teleangiectasias, capillary and venous malformations, poikiloderma of Civatte, and infantile hemangiomas [31-33]. This noncoherent light emits wavelengths 420-1400 nm. Using filters, it emits the required wavelength to target specific chromophores and improve penetration, thereby minimizing energy absorption by other chromophores. The advantages of the IPL system include lower cost, versatility to target multiple chromophores, flexible parameters with less complexity, and fewer side effects [6].
IPL treatments can result in adverse events such as blisters, dyspigmentation, and scar formation. Hyperpigmentation is usually reversible, but hypopigmentation may be permanent [34] as a result of thermal destruction of melanocytes. Incorrect patient selection, i.e., skin color or ethnicity, is an important reason for such adverse effects as there may be variations in melanin content in different people.

Better visualization of blood flow and vessel dimensions may help to improve the results of treatment with IPL [35].

In a prospective randomized study, three 540-nm-wavelength IPL treatments at 4-week interval were tested for telangiectasia in latestage rosacea with positive results for anti-mite therapy of Demodex folliculorum. IPL treatment resulted in a significantly higher rate of efficacy as compared to the control group. The recurrence rate at 2-year follow-up was also lower in the IPL group (8.41\% vs $48.33 \%$ ) [36].

Pain during the PDL treatment is one of the major discomforts faced by the patient. Topical anesthesia can be applied before doing PDL and there are certain studies showing that topical anesthesia does not interfere with the efficacy of the treatment and thus does not affect the outcome [37]. Local anesthesia should be avoided because of probable vessel constriction and consequently less target [38].

IPL emitting non-coherent light (515-1200 nm) tested in 140 patients with linear and spider facial telangiectasia had excellent, good, and poor results in $67.1 \%, 30.7 \%$, and $2.1 \%$ of patients, respectively. There were no major safety concerns in patients treated with IPL [39]. In another study, Chinese patients $(n=35)$ with facial or hand telangiectasia secondary to burns who underwent two to six courses of IPL (wavelength 560-615 nm) over 3-5 weeks had significant reduction in the blood flow in telangiectasia [40].

Out of 30 patients with facial telangiectasia who underwent five sessions of treatment with narrow-band intense pulsed light (500-600 nm) at 4-week interval, 27 (90\%) had more than 50\% clearance post-treatment. Out of 30 cases, eight (27\%) had more than $75 \%$ clearance. At 6-month follow-up, 30\% had recurrence. Only minor side effects were observed [41]. 
A case report suggests the possibility of using IPL in chronic facial erythema and rosacea of the face in systemic lupus erythematosus [42].

\section{Comparison of IPL Versus PDL}

Some investigators have compared the results of IPL versus PDL in facial erythema and telangiectasia [43-48]. In 15 subjects with bilateral facial erythema treatment with IPL was compared versus PDL. On one half, IPL was used and on the contralateral side PDL was given to treat the erythema. The results were evaluated at 30 and 90 days. There was significant improvement in the erythema at the 90th day. There was equal efficacy of IPL and PDL using no purpuric settings [43]. Neuhaus et al. performed a spilt-face study for treating erythematotelangiectatic rosacea comparing 595-nm PDL with $560-n m$ filter of IPL using nonpurpuragenic settings and this study also demonstrated equal efficacy of both IPL and PDL in treating facial erythema [44]. In a study involving nine patients no major differences were noted in efficacy in terms of reducing the melanin or the erythema index [45]. In another study, 16 subjects with facial telangiectasia underwent randomized treatments in the split-face method. These patients received up to two treatments 1 month apart. PDL (595 nm with $10-$ or $7-\mathrm{mm}$ spot; fluence range $8.1-14.5 \mathrm{~J} / \mathrm{cm}^{2}$, and 10 or $40 \mathrm{~ms}$ pulse width) was applied on one side whereas IPL (500-670 and 870-1200 nm, $10 \mathrm{~mm} \times 15 \mathrm{~mm}$ spot, fluence range $34-70 \mathrm{~J} /$ $\mathrm{cm}^{2}, 10$ or $100 \mathrm{~ms}$ pulse width) was applied on the other side of the face. In this study, IPL resulted in similar safety and efficacy as that of PDL [46]. In 2017, Gao and colleagues reported results in facial telangiectasia in a retrospective study involving 416 patients treated with two sessions of either PDL $595 \mathrm{~nm}\left(9-12 \mathrm{~J} / \mathrm{cm}^{2}\right)$, MaxG (500-670 nm and 870-1200 nm, 30-46 J/ $\left.\mathrm{cm}^{2}\right)$, IPL $\left(560-1200 \mathrm{~nm}, 18-24 \mathrm{~J} / \mathrm{cm}^{2}\right), \quad$ M22 $560\left(560-1200 \mathrm{~nm}, 15-18 \mathrm{~J} / \mathrm{cm}^{2}\right)$, or M22 590 $\left(590-1200 \mathrm{~nm}, 15-20 \mathrm{~J} / \mathrm{cm}^{2}\right)$ given 6 weeks apart. Almost all patients in PDL $595 \mathrm{~nm}$ or MaxG groups showed significant improvements or nearly complete clearance of lesions whereas $41-57 \%$ patients in the other three groups had similar improvement. Similarly, the same two treatments had significantly better vessel clearance [47]. In 2020, in a retrospective study, Gao et al. reported results of two sessions of PDL $(595 \mathrm{~nm})$ in comparison with two sessions IPL in 160 patients with facial telangiectasia divided into four study groups: PDL (595 nm), IPL with M22 vascular filter $(530-650 \mathrm{~nm}$ and 900-1200 nm), M22 560 (560-1200 nm), and M22 590 (590-1200 nm). All study groups showed significant improvement. Better results were seen with PDL (595 nm) and IPL vascular filter (530-650 $\mathrm{nm}$ and 900-1200 nm) than the other two groups [48].

\section{KTP and Yellow (577 nm) Lasers}

Copper bromide lasers have been reported to be effective in teleangiectasia and facial erythema; however, the technology is very sensitive. KTP lasers of $532 \mathrm{~nm}$ deliver reliable effective results with mostly robust and durable devices. In a split-face, single-blind, controlled, comparison study, both the 595-nm and the 532-nm pulsed lasers were highly effective in the treatment of facial telangiectasia and redness. The 532-nm KTP laser appears to be more effective but causes more swelling and erythema [49]. Compared to IPL, both large spot KTP and IPL achieved marked improvement in vascular and pigmented lesions in one session. The KTP laser caused slightly more discomfort and edema than the IPL. On the other hand, the KTP laser was faster, and more ergonomically flexible [50].

Because of its ability to specifically target oxyhemoglobin, the 577-nm pro-yellow laser has been deemed ideal for vascular lesions by having an additional advantage over the copper bromide laser (composed of $90 \%$ yellow light and $10 \%$ green light) of minimizing the risk of hyperpigmentation in patients of darker skin types. In addition, it does not require an expensive dye kit as in PDL, nor a cooling device or coupling gel such as in IPL. Kapicioglu et al. [51] showed the efficacy and safety of the 577 -nm pro-yellow laser in erythemotelangiectatic rosacea, facial erythema, and facial telangiectasias in 40 patients treated for one to 
four sessions at 4 -week intervals, at $16-28 \mathrm{~J} / \mathrm{cm}^{2}$ and $6-\mathrm{mm}$ spot mode. A study by Mohamed et al. [52] in 2019 looked into the effectiveness of the 577-nm laser for papulopustular rosacea, facial telangiectasia, and facial erythema, with the most success with lowest number of sessions for facial erythema rather than for facial telangiectasias $(63.6 \%$ of cases with excellent results) using a fluence between 12 and $16 \mathrm{~J} / \mathrm{cm}^{2}$ with pulse duration from 20 to $26 \mathrm{~ms}$, and was done at 1-month intervals for a maximum of five sessions.

In a recent study [53], the 577-nm highpower optically pumped semiconductor laser (HOPSL) has shown promising results for facial erythema using the scanner handpiece, $1-\mathrm{mm}$ spot size, $80 \%$ coverage, $12-15 \mathrm{~J} / \mathrm{cm}^{2}, 30 \mathrm{~ms}$, two passes for three sessions at 1-month intervals.

\section{CONCLUSIONS}

Facial erythema and telangiectasia, in general, respond poorly to treatment. Vascular laser results in heating within dermal blood vessels and reduction of blood vessel diameter. Available evidence suggests that IPL and PDL are both effective in treating erythema. Important parameters for effective results with lasers include wavelength, pulse duration, and fluence. Multiple laser systems with different wavelengths are shown to be effective in the treatment of vascular lesions. There are not many large randomized trials comparing these PDL and IPL therapies. Larger studies are required to establish efficacy of IPL in comparison with PDL.

\section{ACKNOWLEDGEMENTS}

Funding. No funding or sponsorship was received for this study or publication of this article.

Authorship. All named authors meet the International Committee of Medical Journal Editors (ICMJE) criteria for authorship for this article, take responsibility for the integrity of the work as a whole, and have given their approval for this version to be published.

Author Contributions. Vani Yepuri: writing and revising the manuscript. Anant Patil: review and revising the manuscript. Klaus Fritz: review and revising the manuscript. Carmen Salavastru: review and revising the manuscript. George Kroumpouzos: review and revising the manuscript. Steven Paul Nisticò: review and revising the manuscript. Domenico Piccolo: review and revising the manuscript. Ahmed Sadek: review and revising the manuscript. Ashraf Badawi: review and revising the manuscript. Martin Kassir: review and revising the manuscript. Michael H. Gold: review and revising the manuscript. Stephan Große-Büning: review and revising the manuscript. Stephan Grabbe: review and revising the manuscript. Mohamad Goldust: conception, writing, review and revising the manuscript.

Disclosures. Prof. Michael H. Gold has following conflict of interest: Aerolase, Aesthetic Management Partners (AMP), Aesthetic Bio Medical, Alastin, Allergan, Almirall, Bausch Health, BrandMD, Croma, Dermaroller, Endo Aesthetics, EndyMed, Galderma, Hugel, Invasix/InMode, Johnson \& Johnson, Joylux, Leo Pharm, Lumenis, Merz, MTF, NanoPass, Neauvia, Neostrata, Novartis, Nutrafol, Pierre Fabre / Glytone, Revision, Rohrer Aesthetic, Sciton, Sensus, Senté, Skinbetter Science, SkinCeuticals, Skin Medica, Sofwave, Soliton, Stratpharma, Suneva, Venus Concept, VYNE, Zergio. Vani Yepuri, Anant Patil, Klaus Fritz, Carmen Salavastru, George Kroumpouzos, Steven Paul Nisticò, Domenico Piccolo, Ahmed Sadek, Ashraf Badawi, Martin Kassir, Michael H Gold, Stephan Große-Büning, and Stephan Grabbe have nothing to disclose.

Compliance with Ethics Guidelines. This article is based on previously conducted studies and does not contain any new studies with human participants or animals performed by any of the authors. 
Data Availability. Data sharing is not applicable to this article as no datasets were generated or analyzed during the current study.

Open Access. This article is licensed under a Creative Commons Attribution-NonCommercial 4.0 International License, which permits any non-commercial use, sharing, adaptation, distribution and reproduction in any medium or format, as long as you give appropriate credit to the original author(s) and the source, provide a link to the Creative Commons licence, and indicate if changes were made. The images or other third party material in this article are included in the article's Creative Commons licence, unless indicated otherwise in a credit line to the material. If material is not included in the article's Creative Commons licence and your intended use is not permitted by statutory regulation or exceeds the permitted use, you will need to obtain permission directly from the copyright holder. To view a copy of this licence, visit http:// creativecommons.org/licenses/by-nc/4.0/.

\section{REFERENCES}

1. Steinhoff M, Schmelz M, Schauber J. Facial erythema of rosacea-aetiology, different pathophysiologies and treatment options. Acta Derm Venereol. 2016;96:579-86.

2. Cline A, McGregor SP, Feldman SR. Medical management of facial redness in rosacea. Dermatol Clin. 2018;36:151-9.

3. Del Rosso JQ. Management of facial erythema of rosacea: what is the role of topical $\alpha$-adrenergic receptor agonist therapy? J Am Acad Dermatol. 2013;69(6 Suppl 1):S44-56.

4. Wilkin JK. Why is flushing limited to a mostly facial cutaneous distribution? J Am Acad Dermatol. 1988;19:309-13.

5. Izikson L, English JC 3rd, Zirwas MJ. The flushing patient: differential diagnosis, workup, and treatment. J Am Acad Dermatol. 2006;55:193-208.

6. Kapicioglu Y, Sarac G, Cenk H. Treatment of erythematotelangiectatic rosacea, facial erythema, and facial telangiectasia with a 577-nm pro-yellow laser: a case series. Lasers Med Sci. 2019;34:93-98.
7. Ash C, Town G, Whittall R, Tooze L, Phillips J. Lasers and intense pulsed light (IPL) association with cancerous lesions. Lasers Med Sci. 2017;32: 1927-33.

8. Anderson RR, Parrish JA. Selective photothermolysis: precise microsurgery by selective absorption of pulsed radiation. Science. 1983;220:524-7.

9. Husain Z, Alster TS. The role of lasers and intense pulsed light technology in dermatology. Clin Cosmet Investig Dermatol. 2016;9:29-40.

10. Jasim ZF, Woo WK, Handley JM. Long-pulsed (6$\mathrm{ms}$ ) pulsed dye laser treatment of rosacea-associated telangiectasia using subpurpuric clinical threshold. Dermatol Surg. 2004;30:37-40.

11. Goldberg DJ, Sciales CW. Pyogenic granuloma in children. Treatment with the flashlamp-pumped pulsed dye laser. J Dermatol Surg Oncol. 1991;17: 960-2.

12. Marchell N, Alster TS. Successful treatment of cutaneous Kaposi's sarcoma by the $585-\mathrm{nm}$ pulsed dye laser. Dermatol Surg. 1997;23:973-5.

13. Wheeland RG, Applebaum J. Flashlamp-pumped pulsed dye laser therapy for poikiloderma of Civatte. J Dermatol Surg Oncol. 1990;16:12-6.

14. Kelly A, Pai A, Lertsakdadet B, Choi B, Kelly KM. Microvascular effects of pulsed dye laser in combination with oxymetazoline. Lasers Surg Med. 2020;52:17-22.

15. Suggs AK, Macri A, Richmond H, Munavalli G, Friedman PM. Treatment of erythematotelangiectatic rosacea with pulsed-dye laser and oxymetazoline $1.0 \%$ cream: a retrospective study. Lasers Surg Med. 2020;52:38-43.

16. Clark SM, Lanigan SW, Marks R. Laser treatment of erythema and telangiectasia associated with rosacea. Lasers Med Sci. 2002;17:26-33.

17. Fogelman JP, Stevenson ML, Ashinoff R, Soter NA. Idiopathic flushing with dysesthesia: treatment with the $585 \mathrm{~nm}$ pulsed dye laser. J Clin Aesthetic Dermatol. 2015;8:36-41.

18. Alam M, Voravutinon N, Warycha M, et al. Comparative effectiveness of nonpurpuragenic 595-nm pulsed dye laser and microsecond 1064-nm neodymium:yttrium-aluminum-garnet laser for treatment of diffuse facial erythema: a double-blind randomized controlled trial. J Am Acad Dermatol. 2013;69:438-43.

19. Park KY, Ko EJ, Seo SJ, Hong CK. Comparison of fractional, nonablative, 1550-nm laser and 595-nm pulsed dye laser for the treatment of facial 
erythema resulting from acne: a split-face, evaluator-blinded, randomized pilot study. J Cosmet Laser Ther. 2014;16:120-3.

20. Uebelhoer NS, Bogle MA, Stewart B, Arndt KA, Dover JS. A split-face comparison study of pulsed 532-nm KTP laser and 595-nm pulsed dye laser in the treatment of facial telangiectasias and diffuse telangiectatic facial erythema. Dermatol Surg. 2007;33:441-8.

21. Ross M, Watcher MA, Goodman MM. Comparison of the flashlamp pulsed dye laser with the argon tunable dye laser with robotized handpiece for facial telangiectasia. Lasers Surg Med. 1993;13: 374-8.

22. Madan V, Ferguson J. Using the ultra-long pulse width pulsed dye laser and elliptical spot to treat resistant nasal telangiectasia. Lasers Med Sci. 2010;25:151-4.

23. Tan E, Vinciullo C. Pulsed dye laser treatment of spider telangiectasia. Australas J Dermatol. 1997;38: 22-5.

24. Halachmi S, Israeli H, Ben-Amitari D, Lapidoth M. Treatment of the skin manifestations of hereditary hemorrhagic telangiectasia with pulsed dye laser. Lasers Med Sci. 2014;29:321-4.

25. Harries PG, Brockbank MJ, Shakerspeare PG, Carruth JA. Treatment of hereditary haemorrhagic telangiectasia by the pulsed dye laser. J Laryngol Otol. 1997;111:1038-41.

26. Broska P, Martinho E, Goodman MM. Comparison of the argon tunable dye laser with the flashlamp pulsed dye laser in treatment of facial telangiectasia. J Dermatol Surg Oncol. 1994;20:749-53.

27. Tanghetti E, Sherr E. Treatment of telangiectasia using the multi-pass technique with the extended pulse width, pulsed dye laser (Cynosure V-Star). J Cosmet Laser Ther. 2003;5:71-5.

28. Alam M, Dover JS, Arndt KA. Treatment of facial telangiectasia with variable-pulse high-fluence pulsed-dye laser: comparison of efficacy with fluences immediately above and below the purpura threshold. Dermatol Surg. 2003;29:681-4.

29. Hammes S, Raulin C. Evaluation of different temperatures in cold air cooling with pulsed-dye laser treatment of facial telangiectasia. Lasers Surg Med. 2005;36:136-40.

30. Kwon WJ, Park BW, Cho EB, Park EJ, Kim KH, Kim KJ. Comparison of efficacy between long-pulsed Nd: YAG laser and pulsed dye laser to treat rosacea-associated nasal telangiectasia. J Cosmet Laser Ther. 2018;20:260-4.
31. Goldman MP. Treatment of benign vascular lesions with the Photoderm VL high-intensity pulsed light source. Adv Dermatol. 1997;13:503-21.

32. Raulin C, Schroeter CA, Weiss RA, Keiner M, Werner $\mathrm{S}$. Treatment of port-wine stains with a noncoherent pulsed light source: a retrospective study. Arch Dermatol. 1999;135:679-83.

33. Jay H, Borek C. Treatment of a venous-lake angioma with intense pulsed light. Lancet. 1998;351(9096): 112. https://doi.org/10.1016/S0140-6736(05) 78149-6.

34. Polla LL, Tan OT, Garden JM, Parrish JA. Tunable pulsed dye laser for the treatment of benign cutaneous vascular ectasia. Dermatologica. 1987;174: $11-7$.

35. Taudort EH, Olsen J, Andersen PL, Bouazzi D, Jemec GBE. Dynamic optical coherence tomography imaging of telangiectasia prior to intense pulsed light treatment-an opportunity to target treatment? Lasers Surg Med. 2021;53:212-8.

36. Luo Y, Luan X-L, Zhang J-H, Wu L-X, Zhou N. Improved telangiectasia and reduced recurrence rate of rosacea after treatment with $540 \mathrm{~nm}$-wavelength intense pulsed light: a prospective randomized controlled trial with a 2-year follow-up. Exp Ther Med. 2020;19:3543-50.

37. Chunharas C, Boen M, Alhaddad M, Wu DC. The efficacy of pulsed dye laser pretreated with or without local anesthetic on patients presenting with erythema of face, neck, chest, and extremities. Lasers Surg Med. 2020;52:307-14.

38. Stier MF, Glick SA, Hirsch RJ. Laser treatment of pediatric vascular lesions: port wine stains and hemangiomas. J Am Acad Dermatol. 2008;58: 261-85.

39. Retamar RA, Chames C, Pellerano G. Treatment of linear and spider telangiectasia with an intense pulsed light source. J Cosmet Dermatol. 2014;3: 197-290.

40. Li N, Han J, Hu D, et al. Intense pulsed light is effective in treating postburn hyperpigmentation and telangiectasia in Chinese patients. J Cosmet Laser Ther. 2018;20:436-41.

41. Gan H, Yue B, Wang Y, Lu Z. Treatment of facial telangiectasia with narrow-band intense pulsed light in Chinese patients. J Cosmet Laser Ther. 2018;20:442-6.

42. Levy JL. Intense pulsed light treatment for chronic facial erythema of systemic lupus erythematosus: a case report. J Cutan Laser Ther. 2000;2:195-8. 
43. Handler MZ, Bloom BS, Goldberg DJ. IPL vs PDL in treatment of facial erythema: a split-face study. J Cosmet Dermatol. 2017;16:450-3.

44. Neuhaus IM, Zane LT, Tope WD. Comparative efficacy of nonpurpuragenic pulsed dye laser and intense pulsed light for erythematotelangiectatic rosacea. Dermatol Surg. 2009;35:920-8.

45. Kim BY, Moon HR, Ryu HJ. Comparative efficacy of short-pulsed intense pulsed light and pulsed dye laser to treat rosacea. Cosmet Laser Ther. 2019;21: 291-6.

46. Tanghetti EA. Split-face randomized treatment of facial telangiectasia comparing pulsed dye laser and an intense pulsed light handpiece. Lasers Surg Med. 2012;44:97-102.

47. Gao L, Gao N, Song W, et al. A retrospective study on efficacy of pulsed dye laser and intense pulsed light for the treatment of facial telangiectasia. J Drugs Dermatol. 2017;16:1112-6.

48. Gao L, Qu H, Gao N, et al. A retrospective analysis for facial telangiectasia treatment using pulsed dye laser and intense pulsed light configured with different wavelength bands. J Cosmet Dermatol. 2020;19:88-92.
49. Uebelhoer N, Bogle M, Stewart B, Arndt KA, Dover JS. A split-face comparison study of pulsed 532-nm KTP laser and 595-nm pulsed dye laser in the treatment of facial telangiectasias and diffuse telangiectatic facial erythema. Dermatol Surg. 2007;33:441-8.

50. Butler EG, McClellan SD, Ross EV. Split treatment of photodamaged skin with KTP 532 nm laser with 10 mm handpiece versus IPL: a cheek-to-cheek comparison. Lasers Surg Med. 2006;38:124-8.

51. Kapicioglu Y, Sarac G, Cenk H. Treatment of erythematotelangiectatic rosacea, facial erythema, and facial telangiectasia with a 577-nm pro-yellow laser: a case series. Lasers Med Sci. 2019;34:93-8.

52. Mohamed E-E, Mohamed Tawfik K, Hassan AW. Successful treatment of facial vascular skin diseases with a 577-nm pro-yellow laser. J Cosmet Dermatol. 2019;18:1675-9.

53. Wanitphakdeedecha R, Cembrano KAG, Ungaksornpairote $C$, et al. The efficacy and safety of a 577-nm high-power optically pumped semiconductor laser in the treatment of postacne erythema. J Cosmet Dermatol. 2020;00:1-6. 